\title{
BMJ Open Quality Embedding best transfusion practice and blood management in neonatal intensive care
}

Cindy J Flores (1) , ${ }^{1}$ Anil Lakkundi, ${ }^{2}$ Joanne Mclntosh, ${ }^{2}$ Peter Freeman, ${ }^{3}$ Amanda Thomson, ${ }^{1}$ Ben Saxon, ${ }^{1,4}$ Justine Parsons, ${ }^{2}$ Tracey Spigiel, ${ }^{1}$ Sarah Milton, ${ }^{5}$ Bryony Ross ${ }^{6,7}$

To cite: Flores CJ, Lakkundi A, McIntosh J, et al. Embedding best transfusion practice and blood management in neonatal intensive care. BMJ Open Quality 2020;9:e000694. doi:10.1136/ bmjoq-2019-000694

- Additional material is published online only. To view please visit the journal online (http://dx.doi.org/10.1136/ bmjoq-2019-000694)

Received 26 March 2019 Revised 2 December 2019 Accepted 5 December 2019
Check for updates

(C) Author(s) (or their employer(s)) 2020. Re-use permitted under CC BY-NC. No commercial re-use. See rights and permissions. Published by BMJ.

For numbered affiliations see end of article.

Correspondence to

Dr Cindy J Flores;

cflores@redcrossblood.org.au

\section{ABSTRACT}

Background Transfusion is a common procedure for neonates receiving intensive care management. Recognising a paucity of patient blood management (PBM) programmes in neonates, we aimed to embed blood management and best transfusion principles in the neonatal intensive care unit (NICU) by aligning local policies, providing targeted education and partnering with parents.

Methods Practice-based evidence for clinical practice improvement (PBE-CPI) methodology was used. Previous hospital accreditation audits were reviewed and a neonate-specific transfusion audit was developed. Audit was performed at baseline and repeated following the intervention period. NICU clinicians received targeted education in obtaining informed consent, prescription and safe administration of blood components during a 'Blood Month' awareness period. A neonate-specific parent handout about transfusion was developed in partnership with parents. A pilot video demonstrating a shared consent discussion was also developed to assist in the consent process. Parents' knowledge, concerns and feedback regarding transfusion practice was sought at baseline (survey) and on project completion (experience trackers).

Results Neonate-specific baseline transfusion audit showed inconsistent consent, monitoring and documentation processes in neonatal transfusions. Post-targeted education audit showed improvement in these parameters. The targeted PBM and transfusionrelated education delivered during 'Blood Month' was well-received by staff. Parents' feedback about the NICU transfusion consenting process was consistently positive. NICU medical and nursing clinicians $(n=25)$ surveyed agreed that the parent handout was well set out, easy to understand and recommended that it be used to complement practice.

Conclusion PBE-CPI tools aligned with Australian PBM guidelines for clinicians and parents were well-accepted by clinical stakeholders and were associated with practice improvement in PBM awareness and transfusion consent processes. This PBE-CPI project developed NICU-specific consent information, not previously available, by partnering with parents to ensure quality of care in transfusion practice. Adoption of this also helps to meet accreditation for Australian Blood Management Standards. These strategies and tools translate readily into other NICUs to embed and support best PBM and transfusion practice.

\section{INTRODUCTION}

Blood transfusion is a common procedure for neonates requiring intensive care management, particularly among preterm neonates. In New South Wales, neonatal transfusion occurs in about 5.4 per 1000 births and transfusion rates for red blood cells remain constant at $4.8 \%$ per $1000 .{ }^{1}$ Australian Patient Blood Management (PBM) Guidelines include Module 6 Neonatal and Paediatrics to guide care in this patient group. ${ }^{2}$ While many PBM programmes have been successfully implemented in adults to improve transfusion practice, there is paucity of $\mathrm{PBM}$ programmes specifically targeted for neonates, ${ }^{34}$ especially one that aligns with national PBM Module 6 guidelines.

In Australia, hospitals and health services are accredited against the National Safety and Quality Health Service (NSQHS) Standards, which drive safe and quality systems to improve the quality of health service provision. Standard 7, Blood Management, of the NSQHS Standards focuses on patients who need blood management as part of their care, and management of blood and blood products. ${ }^{5}$ Thus, apart from ensuring the principles of the PBM guidelines are integrated in local policies for patient care, health services must also ensure they meet accreditation requirements for the NSQHS Blood Management Standard.

With the paucity of PBM programmes tailored for neonates, this project aims to ensure best practice in neonatal transfusion based on PBM Module 6 and NSQHS Blood Management Standard by: (1) aligning local policies and practice to provide appropriate use of red cell components, (2) providing PBM and transfusion-related education to neonatal intensive care unit (NICU) clinical staff and (3) partnering with parents regarding transfusion practice in neonates. Changes in practice were evaluated with 
neonate-specific audits at baseline and post-intervention, and with feedback from both parents and clinical staff about the interventions.

\section{METHODS \\ Design}

Practice-Based Evidence for Clinical Practice Improvement (PBE-CPI) ${ }^{6}$ methodology was used in a tertiary regional referral hospital that provides comprehensive specialty care to more than 1100 newborns annually. The PBE-CPI project was led by a multidisciplinary team including representation from neonatology, paediatric haematology, NICU nurse practitioners, NICU nurse educator and the Red Cross Lifeblood. The project began in July 2017 and was completed in March 2018.

\section{Baseline measurement}

Previous audits for NSQHS Standard 7 accreditation were reviewed. These were more designed for adult patients with only some elements translatable to neonatal transfusion practice.

A baseline transfusion practice audit was developed specifically for neonatal red cell transfusion, as it is the most common allogeneic blood component transfused in the NICU. Transfusion episodes during 2016 selected through simple random sampling from a laboratory database were included in the snapshot clinical audit.

Parental knowledge, concerns and feedback regarding transfusion practice were sought at baseline with a survey to identify what they perceived to be important information for parents if their baby required a blood transfusion.
PBE-CPI: 'Blood Month' education interventions phase

The PBE-CPI project aim was to improve safety and quality of transfusion practice through increasing compliance with NSQHS Blood Management Standards on NICUspecific consent processes and transfusion documentation. Targets were:

- $100 \%$ of the consent form signed, witnessed and dated

- $100 \%$ documentation in the following areas of the consent form

- Patient label is available on all pages (1-4)

- Patient weight documented

- Blood component prescription properly completed (type of blood component, volume, rate, any special requirements)

- Blood pack ID number documented

- Pathology level recorded (haemoglobin)

- Reason for transfusion (indication) documented

- Completion of transfusion signed

- $60 \%$ documentation of patient name hand-printed and transfusion checklist completed

A multidisciplinary team reviewed and updated current policies and processes involved in the provision of blood and blood products in the NICU. The updated 'Blood administration and blood products in NICU' policy ensured that all clinical transfusion practices and PBM strategies are in accordance with PBM Module 6 and NSQHS Blood Management Standard.

A targeted neonatal PBM education programme suitable for medical and nursing clinicians was developed in consultation with NICU clinicians. An intensive combination of lectures, practical workshops and laboratory

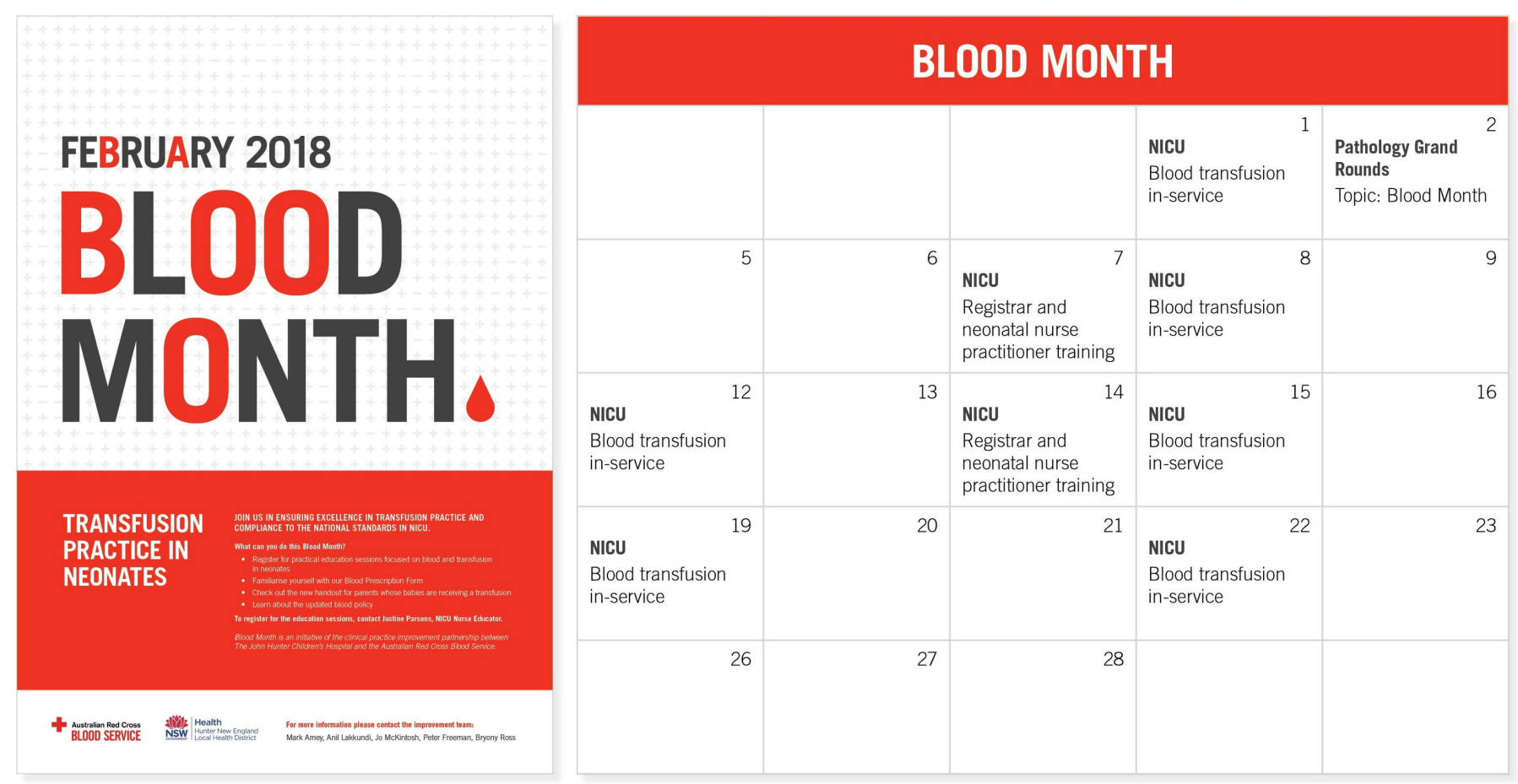

Figure 1 Blood Month calendar of events. NICU, neonatal intensive care unit. 
education was delivered to clinical staff during February 2018: 'Blood Month' (figure 1). Content included:

- An overview of evidence-based transfusion practice in neonates, and evolving areas of research (including transfusion research currently being undertaken within the NICU).

- An outline of the NSQHS Standard 7 Blood Management requirements for the consent, prescription, administration and documentation of blood and blood products (including interactive, interdisciplinary workshops to understand the documentation involved in the transfusion process).

- An overview of the laboratory practices involved for blood and blood products with an emphasis on the additional requirements for neonates.

In partnership with parents and using information from the baseline feedback, a neonate-specific parent handout about transfusion was developed to assist in the informed consent process (online supplementary appendix S1). A pilot video demonstrating a consent discussion was also developed, filmed using an iPhone and edited in-house.

\section{Post-Blood Month feedback}

A post-Blood Month transfusion practice audit was collated and compared with the baseline audit. Neonates who had red cell transfusion episodes during March and April 2018 were identified through convenience sampling from a laboratory database and were included in the snapshot clinical audit.

Feedback from the NICU clinicians regarding the additional transfusion education and neonate-specific parent handout were also collated using a survey.

Parents' feedback regarding transfusion process and the neonate-specific parent handout were sought using iPad experience trackers.

\section{Participants}

Eighty-six out of 195 (44\%) NICU clinicians (9 doctors, 4 neonatal nurse practitioners and 73 nurses) participated in the Blood Month education initiative. Twentyfive NICU clinicians answered the survey, either online or hardcopy, that was circulated to the staff post-Blood Month.

Seventeen parents $(\mathrm{n}=11$ at baseline, $\mathrm{n}=6$ post-Blood Month) provided feedback in this PBE-CPI project. The parents who provided feedback at baseline were randomly selected from NICU parents whose babies are premature and who are at significant risk of receiving a transfusion. Post-Blood Month feedback was collected from consecutive sampling of parents whose babies had a red cell transfusion in the NICU.

All participation was voluntary.

\section{Data analysis}

Data for audits were collected retrospectively from Pathology North's laboratory database and medical charts. $\chi^{2}$ test was performed to compare proportions observed in two samples using MedCalc Software. ${ }^{7}$
Feedback from clinical staff were collected using online SurveyMonkey. Hardcopy returned surveys were manually entered in SurveyMonkey. Baseline parents survey were also collected using SurveyMonkey, facilitated by either NICU nurse practitioner or neonatologist. Descriptive statistics were used to feedback survey results.

Post-Blood Month feedback from parents were collected using iPad patient experienced trackers. Data were entered into an online database and a real-time report was generated using third-party software by Customer Feedback Systems Australasia Pty Ltd.

\section{RESULTS}

\section{Compliance with NSQHS Blood Management Standard 7}

More than 150 units of blood and blood products were prescribed to neonatal patients in 2015 at our tertiary hospital. Previous audits for NSQHS Standard 7 accreditation identified several areas that required improvement in the provision of these products including: completion of consent, recording the indications for transfusion, checking and documenting patient identification and products being transfused, documentation of observations during transfusion, and carer being provided with written information regarding transfusion.

The neonate-specific baseline transfusion audit $(n=59)$ showed there was inconsistent consent documentation with only $10.2 \%$ patient name hand-printed (hospital policy) and $89.8 \%$ with consent signed. There was low procedural documentation with only $47.5 \%$ of patients having their weight documented, $22 \%$ having a transfusion checklist completed (hospital policy is to check every unit) and $30.5 \%$ signed a when the transfusion was complete (table 1). Clinical staff were not aware of any predefined targets for measurement of compliance with the NSQHS Blood Management Standard as the focus was education of staff of key PBM Module 6 recommendations. Following 'Blood Month', the transfusion audit $(n=20)$ showed statistically significant improvements in compliance in transfusion checklist completion ( $\uparrow 63 \%$; $\mathrm{p}<0.0001)$, patient name hand-printed ( $\uparrow 54.8 \%$; $\mathrm{p}<0.0001)$, patient weight document $(\uparrow 47.5 \%$; $\mathrm{p}=0.0002)$ and an almost significant improvement in documenting reason for transfusion ( $\uparrow 20.4 \%$; $\mathrm{p}=0.0512)$. The results also found the number of infants needing increased respiratory support in the 24 hours post-transfusion (using a peak level of respiratory support recorded in the observation chart as the measure) decreased by $8.7 \%$; however, this was not significant. There were no transfusion errors recorded on the blood transfusion prescription and consent form.

The potential causes contributing to the inconsistent consent, monitoring and documentation processes in neonatal transfusion (figure 2) were discussed by the project team. One significant issue identified was the lack of neonatal-specific information available to give to parents during the transfusion consent process. 
Table 1 Clinical audits of compliance (\%) with PBM and the blood and blood product form

\begin{tabular}{|c|c|c|c|c|}
\hline & $\begin{array}{l}\text { July } 2017 \\
\text { (random transfusion } \\
\text { episodes in 2016), } n=59\end{array}$ & $\begin{array}{l}\text { March and April } \\
2018 \\
n=20\end{array}$ & Difference & $\begin{array}{l}\text { P value } \\
(95 \% \mathrm{Cl})\end{array}$ \\
\hline \multicolumn{5}{|l|}{ Consent } \\
\hline Is there a patient label? & $96.5 \%$ & $100 \%$ & $\uparrow 3.5 \%$ & $>0.05$ \\
\hline Patient name hand-printed & $10.2 \%$ & $65 \%$ & $\uparrow 54.8 \%$ & $<0.0001^{*}$ \\
\hline Consent signed, witnessed and dated & $89.5 \%$ & $100 \%$ & $\uparrow 10.5 \%$ & $>0.05$ \\
\hline \multicolumn{5}{|l|}{ Appropriateness } \\
\hline Is it within current NICU transfusion guideline? & $83.1 \%$ & $95 \%$ & $\uparrow 11.9 \%$ & $>0.05$ \\
\hline \multicolumn{5}{|l|}{ Procedure } \\
\hline Patient label & $96.6 \%$ & $100 \%$ & $\uparrow 3.4 \%$ & $>0.05$ \\
\hline Patient weight documented & $47.5 \%$ & $95 \%$ & $\uparrow 47.5 \%$ & $0.0002^{*}$ \\
\hline Blood component prescription properly completed & $91.5 \%$ & $100 \%$ & $\uparrow 8.5 \%$ & $>0.05$ \\
\hline Pack ID number documented & $96.6 \%$ & $100 \%$ & $\uparrow 3.4 \%$ & 0.05 \\
\hline Reason for transfusion documented & $74.6 \%$ & $95 \%$ & $\uparrow 20.4 \%$ & 0.0512 \\
\hline Check/tick list completed & $22.0 \%$ & $85 \%$ & $\uparrow 63 \%$ & $<0.0001^{*}$ \\
\hline Completion of transfusion signed & $30.5 \%$ & $65 \%$ & $\uparrow 34.5 \%$ & $0.0067^{\star}$ \\
\hline \multicolumn{5}{|l|}{ Outcomes } \\
\hline $\begin{array}{l}\text { No of neonates who had an increased level of } \\
\text { respiratory support in the } 24 \text { hours period post- } \\
\text { transfusion }\end{array}$ & $23.7 \%$ & $15 \%$ & $\downarrow 8.7 \%$ & $>0.05$ \\
\hline Necrotising enterocolitis occurred? & Nil & Nil & & \\
\hline \multicolumn{5}{|l|}{ Demographics } \\
\hline \multicolumn{5}{|l|}{ Gestational age } \\
\hline$<29$ weeks & 35 & 5 & & \\
\hline 29-35 weeks & 13 & 10 & & \\
\hline$>35$ weeks & 11 & 15 & & \\
\hline
\end{tabular}

*Statistically significant using $\chi^{2}$ test to compare proportions observed in two samples.

$\mathrm{NICU}$, neonatal intensive care unit; PBM, patient blood management.

Consumer information (parent) handout: babies receiving a blood transfusion-information for parents

Parents $(n=11)$ in the NICU identified what they perceived to be important content for the handout and indicated that the reason for the transfusion and the risks of a transfusion were very important information to be covered

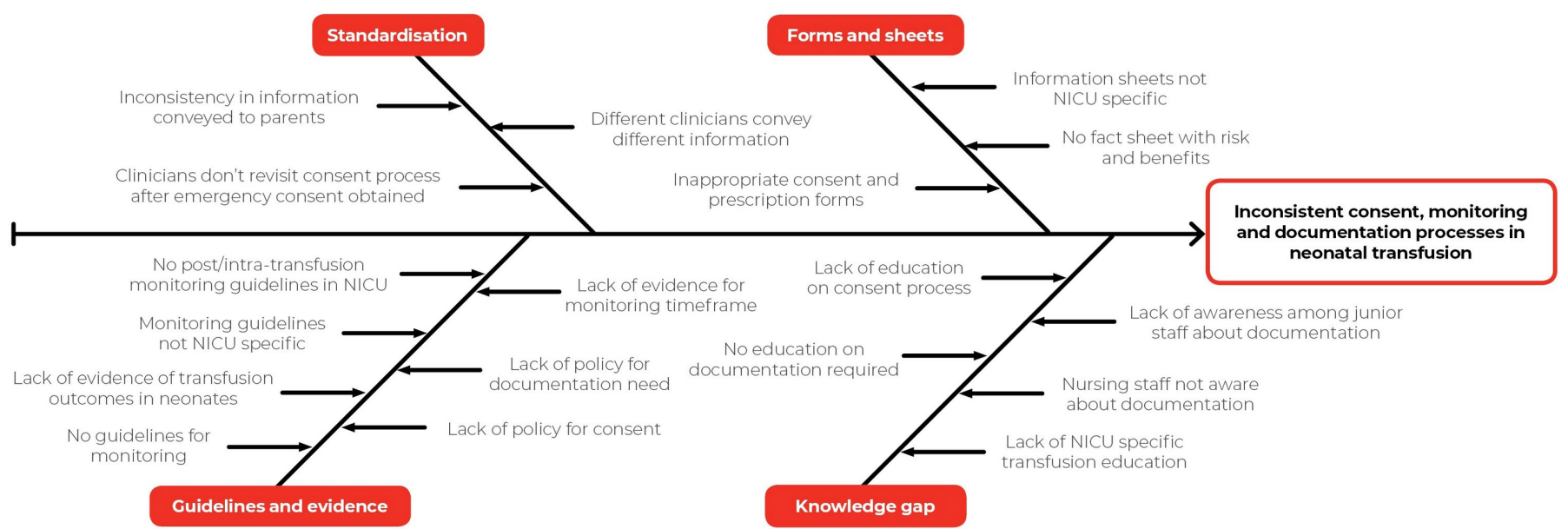

Figure 2 Inconsistencies in the processes of neonatal transfusion. NICU, neonatal intensive care unit. 
A

\begin{tabular}{l|l|l|l|l|l|l|l} 
Key: Very important & 1 & 2 & 3 & 4 & 5 & Very important
\end{tabular}

Q1 Why transfusion may be needed

Q2 What other treatments could be given instead

Q3 What happens if my baby doesn't receive a transfusion

Q4 What are the risks of transfusion

Q5 What are the different parts of blood and what do they do (e.g. red cells, platelets)

Q6 A description of the transfusion procedure

Q7 What will my baby feel during the transfusion

Q8 How safe is the blood supply

Q9 Can parents give blood for their baby instead of from a blood donor

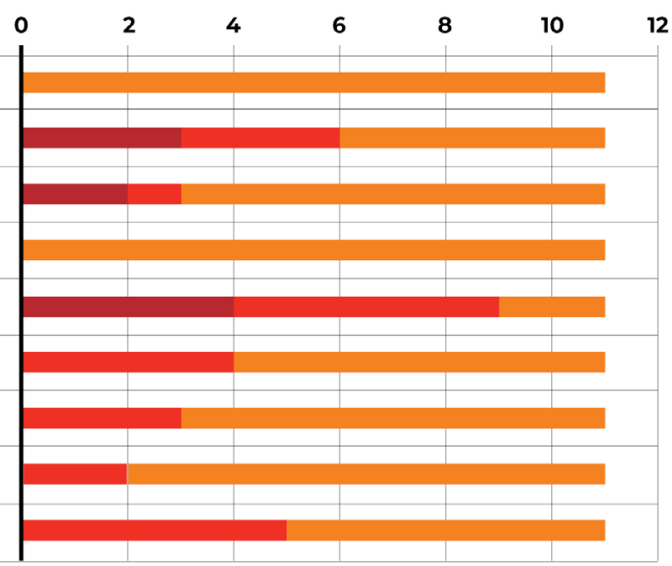

B

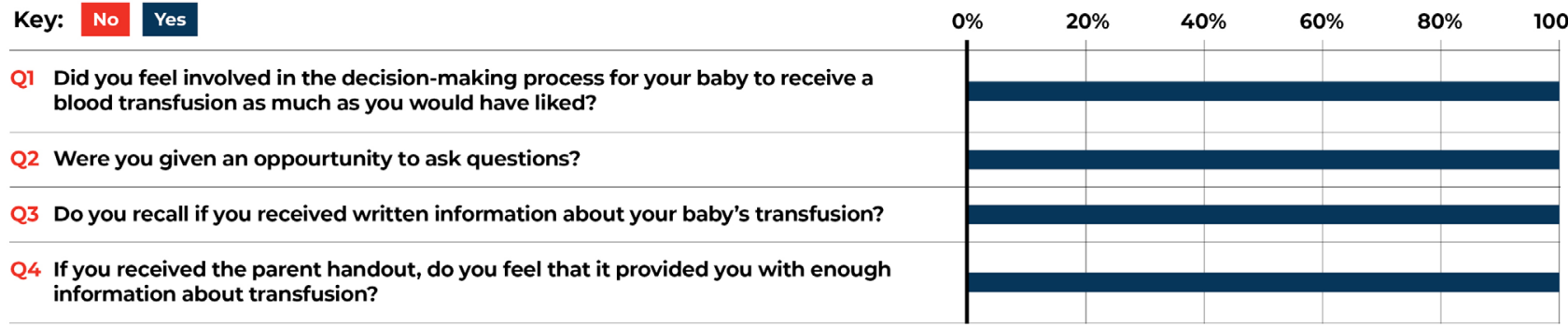

C

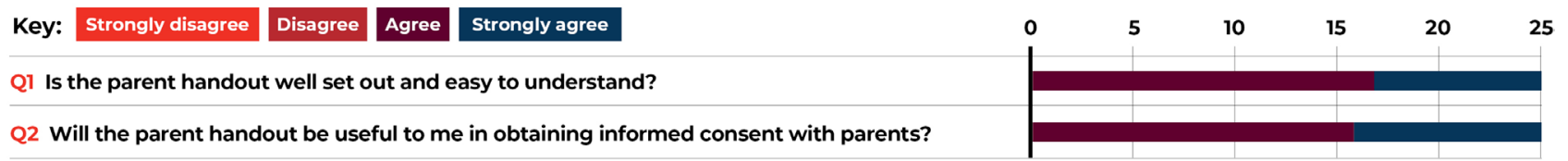

Figure 3 Survey feedback on consumer handout from parents at baseline (A), parents' post-Blood Month (B) and from neonatal intensive care unit clinicians (C).

surveyed agreed that the parent handout was well set out and easy to understand (100\% agree to strongly agree) and will be useful to them in obtaining informed consent with parents (100\% agree to strongly agree). Comments included: "... it is a useful tool, informative for parents in a clear concise way", "easy to read" and "every parent gets the same info". Thus, all surveyed clinicians recommended for the parent handout to be used to complement practice.

\section{'Blood Month' PBM and transfusion education}

Nine education events were delivered in the NICU in February 2018 dubbed as 'Blood Month'. The additional 'Blood Month' education was well-received by clinicians and was seen to be effective in raising awareness about neonatal PBM and transfusion practice, and about the updated 'Blood administration and blood products in NICU' policy. Clinicians reported that Blood Month increased their knowledge about the elements of a transfusion prescription and to be confident in completing the prescription correctly ( $100 \%$ agree to strongly agree) (figure 4). Feedback regarding utility of the in-house pilot video which modelled an 'ideal' informed consent discussion and documentation were not formally sought.
Anecdotal feedback from clinicians regarding the pilot video confirmed that such a tool would be valuable and supported the production of a professional video.

\section{DISCUSSION}

Neonates commonly require transfusion, most often due to anaemia of prematurity. ${ }^{8}$ In 2017, 112 red cell transfusions were administered in the NICU in our hospital. It is essential that processes are in place to ensure safe and appropriate transfusion decision-making, consent, documentation and administration in this vulnerable population. However, in a recent study, Australian and New Zealand neonatal units were found to have significant variation in red cell clinical transfusion practices and local policies. ${ }^{9}$ This PBE-CPI project confirmed the varied practice and inconsistent documentation practices in the NICU. For example, while it is understood that most NICU patients are weighed on admission and most clinicians would frequently use recent weight as a basis for calculation of transfusion volume (unless it is an emergency transfusion at birth), there was initially a low compliance with weight documentation on the blood transfusion prescription and consent form, but the weight 


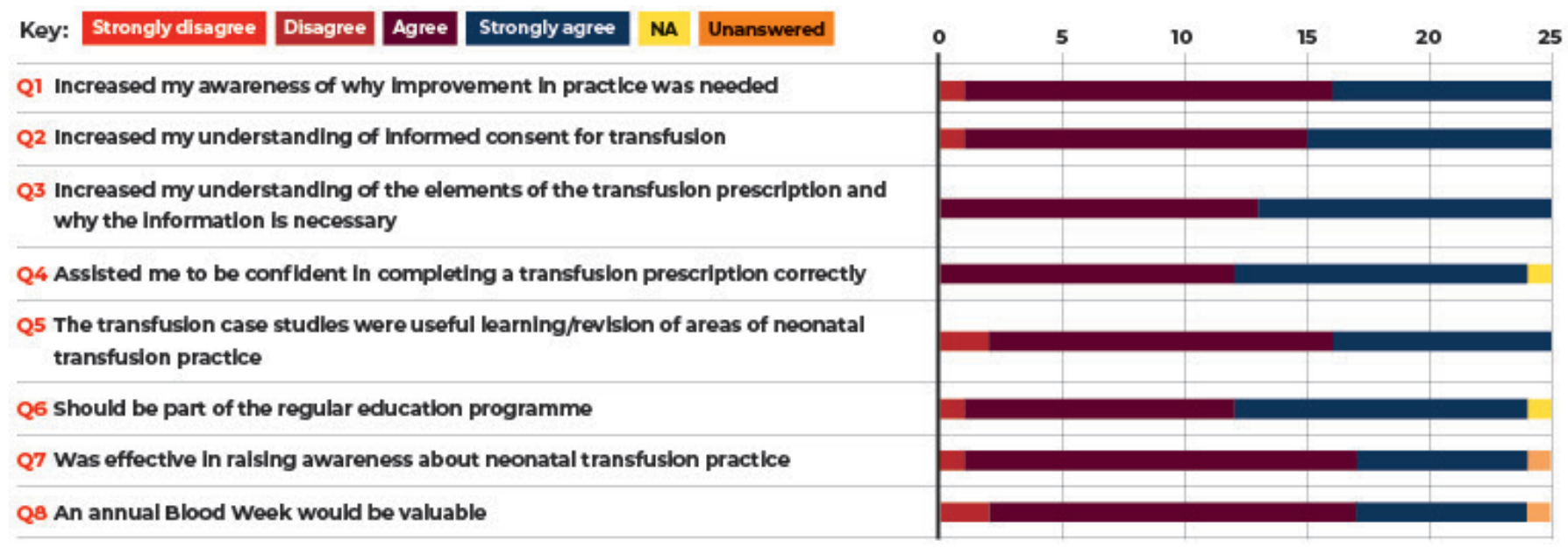

Figure 4 Neonatal intensive care unit clinicians' feedback on Blood Month.

is always documented elsewhere in the medical record. A significant improvement in documentation of weight on this particular transfusion form, as recommended in the guidelines, was seen post-Blood Month education.

A revised local transfusion and blood management 'Blood administration and blood products in NICU' policy, which was launched and communicated during Blood Month, provided updated guidance for our NICU clinicians. We also began to address the paucity of local and international PBM programmes and resources specifically targeted for neonates by making these available for NICU clinical staff. ${ }^{34}$ As a result of this PBE-CPI project, the term PBM, and understanding of its principles, became familiar to our NICU clinical staff. Successful PBM programmes rely on staff education, multidisciplinary engagement and buy-in from clinicians. ${ }^{3}$ Our NICU clinical staff have recognised that implementing the PBM Module 6 guidelines and meeting the NSQHS Blood Management Standard will help ensure the highest quality care, and this was met with improvement in consent processes and blood transfusion documentation.

Patient-centred care is a key element for a neonatal PBM programme. ${ }^{310}$ The new 'Babies receiving a transfusion' parent handout assisted with the transfusion informed consent process and was positively received by both parents and clinical staff. The wider need for such information was confirmed by a major children's hospital in Melbourne, Victoria, who were contacted being keen to use the resource. Following review and minor modifications being integrated, the resource has now been made available nationally to help support best practice. In addition, a professional version of the video 'Transfusion consent for neonates: a shared decision' was produced and available on the Lifeblood website for health professionals. ${ }^{11}$

There are a number of recognised limitations to the results of the project. Clinical practice improvement methodology was used and only one improvement cycle was performed, which involved multiple interventions and a relatively short follow-up. Therefore, it is not possible to determine which of the interventions had the most positive effect. Another limitation is that $\chi^{2}$ tests of proportions is sensitive to sample size and does not take into account multiple variables. Our sample size of combined 79 transfusion episodes out of 112 red cell transfusions in 2017 has a $6.01 \%$ margin of error. Parent sampling may also have been biased with random and consecutive sampling used at a time when it was most suitable for us to interview the parents at a stressful time in their life. There was no attempt to stratify parents and their premature babies who received a red cell transfusion; thus, parent sampling may not be representative of Australian multicultural parents population.

Despite these limitations, sample selection for these process-based audits for PBE-CPI project enabled the project team to measure the whether the NICU processes are aligned with NSQHS Blood Management Standard or PBM Module 6 to facilitate improvement. The PBE-CPI interventions have shown considerable benefit and have been embedded within clinical practice. For example, the parent handout is always included in the NICU blood prescription and consent form so all families receive this information when a transfusion is requested. Repeat audit of practice is planned to determine whether the gains have been maintained while transfusion and PBM has become a part of ongoing NICU education.

To our knowledge, this PBE-CPI project is one of the first attempts to implement a PBM programme in neonatal practice in Australia. The project showed that a combination of neonate-specific PBE-CPI tools, clinician education and parent information aligned with Australian PBM Guidelines and national safety and quality standards were well-accepted by clinical stakeholders. Development of neonate-specific consent information, not previously available, and partnering with parents promoted PBM and best transfusion practice principles, and improved compliance with the NSQHS Blood Management Standard. The study therefore demonstrated the potential to improve PBM and transfusion practice in this vulnerable population. The strategies used and tools developed 
translate readily into other NICUs to support best patient care.

Author affiliations

${ }^{1}$ Clinical Services and Research Division-Transfusion Policy and Education, Australian Red Cross Lifeblood, Adelaide, South Australia, Australia

${ }^{2}$ Neonatal Intensive Care Unit, John Hunter Children's Hospital, Newcastle, New South Wales, Australia

${ }^{3}$ Hunter New England Local Health District, New Lambton, New South Wales, Australia

${ }^{4}$ Women's and Children's Hospital Adelaide, North Adelaide, South Australia, Australia

${ }^{5}$ The Royal Children's Hospital, Melbourne, Parkville, Victoria, Australia

${ }^{6}$ Oncology and Haematology, John Hunter Children's Hospital, Newcastle, New

South Wales, Australia

${ }^{7}$ Calvary Mater Hospital, Newcastle, New South Wales, Australia

Acknowledgements We thank the NICU staff and parents for their support and involvement in the PBE-CPI study. We also thank Hunter New England Local Health District Workforce Quality and Projects for their assistance. Thanks to Anne Kinmonth for coordinating the feedback on the Babies receiving a blood transfusion-information for parents' handout with Blood Management Committee, clinical staff and parents from the Royal Children's Hospital Melbourne. Australian governments fund the Australian Red Cross Lifeblood to provide blood, blood products and services to the Australian community.

Contributors All authors contributed to the design, implementation and review of the PBE-CPI project. BR is the clinical lead with C Flores coordinating the whole PBE-CPI project. CJF, AL, JM, PF, AT and BR were involved in the planning and diagnostic phase of the CPI methodology. AL, PF and BR completed the snapshot clinical audits. SM, TS, CJF and BS drafted and finalised the parents' handout. $\mathrm{JP}$ and JM conducted the interviews with parents. AL, JM, PF, JP, AT and BR implemented Blood Month in the NICU with resources developed and reviewed by $\mathrm{BS}, \mathrm{AT}$, CJF and TS. CJF wrote the first manuscript and is the corresponding author. CJF, BR, PF and AT collated and evaluated the data. All authors provided review of the manuscript, revised the manuscript and agreed on the final version submitted.

Funding The authors have not declared a specific grant for this research from any funding agency in the public, commercial or not-for-profit sectors.

Competing interests None declared.

Patient consent for publication Not required.

Ethics approval The project was approved by the Hunter New England Human Research Ethics Committee (AU201706-07) as low-risk research.
Provenance and peer review Not commissioned; externally peer reviewed.

Data availability statement All data relevant to the study are included in the article.

Open access This is an open access article distributed in accordance with the Creative Commons Attribution Non Commercial (CC BY-NC 4.0) license, which permits others to distribute, remix, adapt, build upon this work non-commercially, and license their derivative works on different terms, provided the original work is properly cited, appropriate credit is given, any changes made indicated, and the use is non-commercial. See: http://creativecommons.org/licenses/by-nc/4.0/.

ORCID iD

Cindy J Flores http://orcid.org/0000-0002-5636-6509

\section{REFERENCES}

1 Bowen JR, Patterson JA, Roberts CL, et al. Red cell and platelet transfusions in neonates: a population-based study. Arch Dis Child Fetal Neonatal Ed 2015;100:F411-5.

2 National Blood Authority (NBA). Patient Blood Management Guidelines: Module 6 - Neonatal and Paediatrics. Canberra, Australia: NBA, 2016.

3 Crighton GL, New HV, Liley HG, et al. Patient blood management, what does this actually mean for neonates and infants? Transfus Med 2018;28:117-31.

4 Goobie SM, Gallagher T, Gross I, et al. Society for the advancement of blood management administrative and clinical standards for patient blood management programs. 4th edition (pediatric version). Pediatric Anesthesia 2019;29:231-6.

5 Australian Commission on Safety and Quality and Health Care. National Safety and Quality Health Service Standards, second edition. Sydney NSW: ACSQHC, 2018.

6 Horn SD, Gassaway J. Practice-Based evidence study design for comparative effectiveness research. Med Care 2007;45:S50-7.

7 MedCalc Software. Comparison of proportions calculator. Available: https://www.medcalc.net/statisticaltests/comparison_of_proportions. php [Accessed 1 Aug 2019].

8 Aher S, Malwatkar K, Kadam S. Neonatal anemia. Semin Fetal Neonatal Med 2008;13:239-47.

9 Saito-Benz M, Sandle ME, Jackson PB, et al. Blood transfusion for anaemia of prematurity: current practice in Australia and New Zealand. J Paediatr Child Health 2019;55:433-40.

10 Goel R, Cushing MM, Tobian AAR. Pediatric patient blood management programs: not just Transfusing little adults. Transfus Med Rev 2016;30:235-41.

11 Australian Red Cross Lifeblood. Resources for neonatal transfusion. Available: www.transfusion.com.au/neonates [Accessed 15 Augt 2019]. 